\title{
Biocomercio y sostenibilidad: análisis en torno a las materias primas de las artesanías en Colombia
}

\section{Biocomerce and sustainabillity: an analysis around raw materials in Colombian handicrafts}

\author{
Luisa Fernanda Casas-Caro ${ }^{1^{*}}$ y Alejandro Lozano $\odot$ \\ *Autor de correspondencia: lfcasascaro@gmail.com \\ Recibido: 1 de mayo de 2018 - Aceptado: 19 de septiembre de 2018
}

1 Fundación Natura, Bogóta D.C.

\section{Resumen}

Palabras clave:

artesanías; manejo sostenible; biocomercio; fibras vegetales

Key words:

handicrafts; sustainable management; biocommerce; plant fibers
Las artesanías colombianas han alcanzado un punto alto en su comercialización, han llegado a todo tipo de mercados e incluso se han posicionado como producto decorativo de estatus; sin embargo, muchos son los vacíos y carencias que se dan en el sector artesanal. Un gran porcentaje de oficios artesanales del país emplea fibras de plantas silvestres y cultivadas como fuentes de materia prima, lo que genera problemáticas ambientales asociadas a esta actividad, acompañadas de conflictos sociales y económicos de igual o mayor magnitud. En este artículo se analiza la labor artesanal bajo la mirada del Biocomercio y la Sostenibilidad; los cuales están enmarcados dentro de la Economía Verde, concepto de uso global que contempla una perspectiva balanceada entre el desarrollo social, ambiental y económico. Como eje de discusión central se tomaron los casos de los oficios artesanales de tejeduría en caña flecha (Gynerium sagitatum (Aubl.) P.Beauv), cestería en esparto (Juncus ramboi Barros), tejeduría en wérregue (Astrocaryum standleyanum L.H.Bailey), aplicación en tamo y cestería en rollo de Guacamayas. Los métodos empleados para el levantamiento y análisis de la información correspondieron al planteamiento de una investigación cualitativa, basada en los principios de la etnografía, desarrollada con herramientas como entrevistas semiestructuradas, recorridos de campo, jornadas de observación participativa y mesas de trabajo entre los investigadores. A lo largo del texto evidenciamos y analizamos de forma integral algunos conflictos relacionados con las materias primas en los oficios artesanales como: falta de visibilidad de cultivadores y recolectores, déficit en el abastecimiento y baja calidad de materia prima, presencia de cuellos de botella en la producción artesanal, falta de legalidad, ausencia de manejo sostenible, carencia de pagos justos y escaso fortalecimiento a recolectores y cultivadores de materias primas, entre otros.

\section{Abstract}

Colombian handicrafts have reach a high point in commerce; attaining different markets and even positioning as a top status decorative product; nevertheless, there are lacks and deficits in the handicraft sector. A high percentage of the handicrafts works made in the country use plant fibers of wild and cultivated plants as raw materials, wich causes environmental problems related to this activity, and also social and economic conflicts of equal or greater magnitude. In this article we analyze the handicraft work through the lens of Biocomerce and Sustainability, which are framed in to the Green Economy, a global concept that offers a balanced perspective of social, environmental and economic development. As main axis for the discussion some cases were analized such as caña flecha a weaving (Gynerium sagitatum (Aubl.) P.Beauv), esparto basketry (Juncus ramboi Barros), wérregue weaving (Astrocaryum standleyanum L.H.Bailey), tamo application and Guacamayas basketry. The methods used for gathering information and further analysis correspond to a qualitative research approach, based on ethnographic principles, developed with tools as semi-structured interviews, field walks, journeys of participative observation and workshops with the researchers. In the text we evidence and analyze in an integral way some of the conflicts related with handicrafts raw material such as: few visibility of growers and harvesters, deficit on the supply and low quality of raw material, bottlenecks in the handicraft production, lack of legality, absence of sustainable. 


\section{Introducción}

En el proceso de manufacturación de todo producto, se contempla el suministro de materia prima como indispensable; del mismo modo, en el proceso de elaboración de una artesanía la disponibilidad de materia prima es crucial. En gran medida las materias primas del sector artesanal dependen del ambiente, pues provienen de recursos naturales; y su cantidad y calidad va a determinar también la cantidad y calidad del producto final. Por supuesto, sin dejar de lado aspectos como la pericia de la mano de obra y la capacidad de producción, entre otros. En Colombia los artesanos tienen problemas de escasez de materia prima y dificultades con su calidad (Artesanías de Colombia, 1998). Bajo este panorama, en la producción de artesanías, las materias primas se convierten en un material primordial para el desarrollo del producto; además, su producción, recolección o transformación se relaciona con dinámicas sociales, culturales y económicas propias de cada oficio.

Al integrarse los ejes económicos, sociales y ambientales, el Biocomercio y la Sostenibilidad en los oficios artesanales cobran importancia. A nivel mundial existen diferentes conceptos sobre desarrollo sostenible; los tres más predominantes son Economía Circular, Economía Verde y Bioeconomía (D’Amato et al., 2017). De acuerdo al análisis de D'Amato et al. (2017), los tres conceptos se diferencian y se relaciona con distintos sectores de la economía, comprendiendo variadas dimensiones. La Economía Circular, se enfoca en procesos urbanos industriales y suele ser usada en China; mientras que el término Bioeconomía se usa más ampliamente en Europa y se basa en el uso de recursos biológicos y su innovación, con prácticas de uso en un contexto de desarrollo rural. Finalmente, la Economía Verde es un concepto de uso más global y actúa como sombrilla de los dos anteriores, pues contempla una perspectiva balanceada entre el desarrollo social y ambiental.

Para el caso de Colombia aplica el concepto de Economía Verde, pues las políticas de gobierno sobre este tema buscan dicho desarrollo equitativo entre los aspectos ambientales, sociales y económicos. En el país este concepto se ha incorporado por medio de los Mercados Verdes y del Biocomercio (Programa Nacional de Biocomercio Sostenible, 2014). En particular muchas artesanías son productos de Biocomercio y en aquellos productos cuya definición no corresponde con el concepto del Biocomercio, también se debe propender por su sostenibilidad. La sostenibilidad consiste en la adopción de objetivos de desarrollo sostenible, especialmente equidad social, eficiencia económica y rendimiento ambiental; para lograr estas metas es necesario hacer cambios fundamentales, y estos cambios son denominados innovación (Elzen et al., 2004). Para Sachs (2002) la sostenibilidad es más amplia, y está compuesta por cinco dimensiones básicas: social, económica, ecológica, cultural y geográfica; todas bien identificadas y soportadas en el sector artesanal ( Moraes, 2010).

En cuanto al Biocomercio, ha sido definido como "el conjunto de actividades de recolección, producción, procesamiento y comercialización de bienes y servicios derivados de la biodiversidad nativa; bajo criterios de sostenibilidad ambiental, social y económica" (Biocomercio Colombia, consultado abril 19 de 2017). Si bien, la elaboración de artesanías no siempre presenta sostenibilidad en estos tres aspectos, sí tiene todo el potencial para desarrollarla. Adicionalmente, diversos aspectos del Biocomercio pueden enlazarse como servicios ecosistémicos de aprovisionamiento, que se vinculan a la política de conservación del país (PGNGIBSE, 2012; Programa Nacional de Biocomercio Sostenible, 2014); de hecho, se reportan estudios en los que el uso sostenible de una palma puede llegar a ser considerado como una estrategia de conservación, no solo para la especie, sino para su ecosistema (Castaño-Arboleda et al., 2007; Bernal y Galeano, 2013; García et al., 2011; García et al., 2013; Isaza y Howard, 2013; García et al., 2015; Torres et al., 2016). El vínculo de las diferentes aristas de la sostenibilidad en las artesanías y la conservación, puede constituirse como todo un escenario por explorar, basado en el uso y manejo de materias primas, el fortalecimiento de la cultura en torno a los oficios artesanales y la comercialización de artesanías como productos de Biocomercio y la actividad de economía complementaria.

De manera complementaria, otro concepto importante de resaltar para el caso de las materias primas son los Productos Naturales No Maderables (PNNM), derivados de los bosques cuyo aprovechamiento se da dentro de ecosistemas naturales (González, 2003); por ejemplo, fibras, semillas, resinas y tintes, entre otros. Vale la pena aclarar que entre los PNNM no se encuentran los productos derivados de plantas cultivadas ni los provenientes de animales domésticos (cachos, cueros, lanas, conchas, pluma); sin embargo, las plantas cultivadas cobran importancia dentro de este análisis, ya que en la producción artesanal también ocupan un importante papel como materias primas. Para el sector artesanal también son relevantes los productos minerales como: arcillas y metales (oro, plata, bronce, cobre, etc.), y un sinfín de productos industriales como: hilos, telas, chaquiras, vidrios, etc. No obstante, el análisis y apoyo que requieren 
las fibras naturales, tintes y maderas cobija otro tipo de necesidades y posibilidades. Se debe tener en cuenta que las materias primas usadas en la producción artesanal tienen diferentes orígenes como: vegetal, animal, mineral e industrial; y que la naturaleza de estos orígenes se vuelve importante para analizar los oficios artesanales, los cuales no deben ser vistos sólo bajo una mirada comercial; ya que el abastecimiento de estas materias primas depende principalmente de prácticas culturales de las comunidades de artesanos y recolectores, además de aspectos ambientales, geográficos y comerciales.

En el país el $83 \%$ de las materias primas empleadas en la elaboración de las artesanías son de origen natural, y en muchos casos las plantas son de origen silvestre. Las materias primas de origen vegetal se emplean en el 57,52 \% de los oficios de tejeduría del país que, sumada a aquellas usadas en el trabajo de talla en madera, conforman el 70,9 $\%$ de las materias primas de uso artesanal en Colombia (Artesanías de Colombia, 1998). Cifras que representan un alto porcentaje, por lo que se hace importante dirigir la mirada hacia estas plantas y las interacciones de las comunidades humanas con ellas.

Asimismo, en Colombia se ha documentado que al menos 114 especies de plantas nativas se emplean para la extracción de fibras vegetales, bien sea como fibras de uso industrial y/o artesanal (Linares et al., 2008). Esto es un reflejo de la riqueza natural y cultural que albergan los oficios artesanales desarrollados en el país; sin embargo, al realizar la revisión de literatura sobre el tema, se hace evidente el poco conocimiento sobre la biología y las tradiciones culturales asociadas a la producción artesanal. Estas diferenciaciones son importantes, pues dan claridad sobre características de las especies y sus posibilidades a nivel de producción y mercado en el marco del Biocomercio.

Con miras a la sostenibilidad, a nivel mundial en diversos estudios se ha evidenciado la relación entre lo ambiental, social y económico; esto con distintos matices en el sector artesanal o en el Biocomercio. Por ejemplo, las fibras de las palmas suelen ser usadas para hacer productos de uso local, pero algunos llegan a tener un gran valor económico y en ciertas áreas su comercio tiene un fuerte impacto en las comunidades locales (Linares et al., 2008; García et al., 2011; Isaza y Howard, 2013). En Etiopía, en el caso del bambú, se muestra cómo el aprovechamiento de Productos Naturales No Maderables (PNNM) tiene un impacto directo e importante en la economía de ciertos grupos sociales; su venta genera en promedio un ingreso en efectivo del $11 \%$, el cual varía entre 3,4 y $38 \%$, y se constituye como un aporte representativo para la economía familiar. Según diferentes investigaciones, en México, Bolivia y Bangladesh, un tercio de las familias entrevistadas informaron la venta de PNNM y su respectivo aporte en dinero efectivo (Kar y Jacobson, 2012; Marshall et al., 2006; Mekonnen et al., 2014).

En Brasil, el estudio de caso de una comunidad de la Amazonía en donde se usa el diseño aplicado a las artesanías como una herramienta para la sostenibilidad, evidencia cómo desde un aspecto particular se pueden abarcar todas las dimensiones de dicha sostenibilidad; llegando a aspectos como la consolidación de redes para integrar la competitividad al territorio, con consecuencias en la mejora de la calidad de vida de los miembros de la comunidad (Moraes, 2010). En México, la actividad artesanal es importante, ya que es desarrollada por un gran número de personas (aproximadamente 12,000,000 de artesanos) (Fondo Nacional para Fomento de las Artesanías, 2016) y mejora la economía del país pues, de acuerdo con la cuenta satélite de la cultura de México (CSCM), las artesanías generan el 20,3\% del producto interno bruto del sector cultural (Haro-Zea et al., 2018). Otro efecto positivo del trabajo artesanal es que genera efectos sociales a nivel de género, debido a que en muchos casos se da una importante participación de las mujeres, tal como ocurre en Chiapas (Haro-Zea et al., 2018) y en Boyacá con la tejeduría de esparto (Lozano et al., 2008). Además, el trabajo artesanal no solo contribuye a la actividad económica al generar empleos directos e indirectos, sino que representa el orgullo de ciertos pueblos, es una tradición con gran valor cultural y social y su preservación ayudará a la conservación de la identidad regional (García et al., 2011; Haro-Zea et al., 2018).

En el presente trabajo se busca mostrar la importancia de las materias primas en los oficios artesanales en Colombia, desde los variados y amplios aspectos que influyen en su sostenibilidad, lo cual incluye aspectos ambientales, culturales y económicos; haciendo énfasis en su uso y manejo. Además, busca evidenciar los cambios de percepción y apreciación sobre el papel que juega la materia prima y analiza cómo esta se utilizó dentro de los diferentes procesos que incluye el sector artesanal.

\section{Materiales y métodos}

La presente investigación está enmarcada dentro del proyecto "Saberes, significados y mercados. Análisis crítico sobre la transformación en el oficio artesanal en Colombia”, cuyo propósito fue analizar los distintos factores y contextos que pueden estar incidiendo en los cambios y trasformaciones en el oficio artesanal y la 
producción de 20 artesanías emblemáticas. De los oficios artesanales investigados, varios eran realizados con materias primas vegetales, la sostenibilidad de cinco de estos fue analizada y dio como resultado este artículo.

El proyecto se desarrolló durante dos años, a partir del 2016 hasta el 2018. El lugar de estudio correspondió a las regiones Andina, Caribe, Amazonia y Pacífico sitios en los que se distribuyen los veinte oficios artesanales; sin embargo, los oficios aquí analizados correspondieron solamente a la región andina, Caribe y Pacífico. El equipo de trabajo del proyecto macro fue un grupo interdisciplinario conformado por antropólogos, biólogos, historiadores y diseñadores; todos con experiencia en el tema de artesanías. Los métodos empleados para el levantamiento y análisis de la información se plantearon según una investigación cualitativa, basada en los principios de la etnografía; se usaron como herramientas entrevistas semiestructuradas, recorridos de campo, jornadas de observación participativa y mesas de trabajo entre los investigadores. Además se llevó a cabo una revisión bibliográfica acompañada de la búsqueda de imágenes en archivos locales-familiares, así como en los archivos de entidades como el Ministerio de Cultura, Proexport y Artesanías de Colombia y tesis académicas.

Finalmente, el ejercicio etnográfico permitió identificar las prácticas en el entorno mismo en el que se producen, establecer los elementos con los que son recreadas, las personas que las desarrollan y trasmiten, registrar los procesos de obtención y manejo de materias primas, la manufactura, las redes de comercialización, los cuellos de botella y los retos a los que se ven enfrentados los artesanos en su contexto.

\section{Resultados y discusión}

La información recolectada se analizó de cara a la sostenibilidad, lo que permitió dilucidar cómo las materias primas posibilitan la intervención en los aspectos sociales, económicos y culturales en el sector artesanal. Como ejes de discusión se tuvieron en cuenta la visibilidad de los actores de la cadena de valor de las artesanías, la importancia de las materias primas en el oficio artesanal, los cuellos de botella o barreras para la producción artesanal asociados a las materias primas y su manejo sostenible.

\section{Cuando el papel de las materias primas era invisible}

No siempre el tema de materias primas ha sido considerado como parte importante para el fortalecimiento del 140 sector artesanal; en muchos casos se daba por hecho el abastecimiento constante, y se desconocían las múltiples variables que entran en juego para obtener un recurso natural permanentemente. Además, era totalmente invisible el papel que desempeñaban cultivadores y recolectores de materias primas, y apoyaba sólo a quienes participaban en el proceso a partir de la transformación.

Luego de realizar búsquedas bibliográficas sobre los procesos artesanales, se hace evidente que hubo un cambio notorio en el abordaje del tema. En un principio, resultó recurrente que las narraciones empezaran con descripciones a partir del punto en el que ya se había comprado o recolectado la materia prima. Esto dejó de lado durante muchos años la investigación y documentación sobre los procesos realizados por quienes constituyen este eslabón en la cadena de valor de las artesanías; hecho que ha llevado a la existencia de un vacío de información sobre esta fase inicial de los procesos artesanales.

En este sentido, también se puede resaltar que "solo hasta la década de los sesenta, el estado colombiano empezó a ver a los artesanos como una fuerza productiva importante para la economía» (Ramírez, 2012). Para estas mismas fechas ya se valoraba la artesanía desde la mirada del potencial que tiene hacia las exportaciones y el creciente turismo, misma óptica que aún se mantiene en muchos contextos. El problema con valorar la artesanía solo desde estos aspectos, es que se esconde la riqueza que hay en los procesos y mecanismos que han construido los grupos humanos que las producen, con todas las complejidades culturales que se desarrollan en torno al oficio artesanal en sí (Ramírez, 2012).

Hacia la siguiente década, los setenta, la producción artesanal se centraba no solo en la manufactura, sino también en la forma de expresión de un pueblo. Desde entonces, Artesanías de Colombia S.A. ha tenido la tensión entre mejorar y conservar las artesanías, "promover al artesano hacia nuevos diseños y a la vez conservar y respetar los diseños tradicionales" (Ramírez, 2012). Si bien, desde lo conceptual hace mucho existía una mínima mirada a la materia prima y al medio ambiente, desde la práctica la empresa hizo énfasis en producción y comercialización, y dejó de lado los temas culturales y ambientales.

El vacío en torno a las materias primas se ha ido llenando lentamente con el trabajo realizado desde hace alrededor de tres décadas, en donde se evidenció la necesidad de tener información sobre el cultivo o recolección; sin embargo, en muchos de los oficios la información aún no es de la misma calidad que la información asociada a las posteriores etapas de producción. Se conocen aspectos 
generales de las prácticas de cultivo y recolección pero no en detalle, lo que tiene implicaciones ambientales sobre las especies proveedoras de fibras, tintes y maderas; e implicaciones económicas y sociales sobre las personas dedicadas a la labor de cultivo y/o recolección. De acuerdo a lo que hemos observado en los trabajos de campo durante varios años, en muchos casos los encargados de hacer la recolección o cultivo de las materias primas no son los artesanos, suelen ser personas dedicadas a diversas labores de campo, como el aprovechamiento económico de estas fibras, tintes o maderas. Este esquema es más recurrente en las comunidades campesinas, pues en comunidades negras e indígenas es más frecuente que una misma persona (o un familiar cercano) vaya en busca de la materia prima y luego realice el trabajo artesanal.

A simple vista, la no identificación de los recolectores y/o artesanos no constituye problema alguno, pero a largo plazo ha hecho que las condiciones de vida de quienes se encargan de esta labor no mejoren de la misma manera como las condiciones de artesanos e intermediarios. Además, se ha generado un uso y aprovechamiento de las plantas proveedoras de fibras y maderas, sin control y sin proyecciones a mejorar.

Con el paso del tiempo, en algunos oficios artesanales se evidenció la necesidad de invertir en el manejo y conservación de la materia prima, ya que para ciertos casos se hizo evidente una problemática en torno a su abastecimiento, en especial para las fibras vegetales. Tal vez la palma de wérregue sea el oficio artesanal que mejor ejemplifica cómo la visión de manejo de materias primas empezó a arraigarse en los proyectos realizados en el sector artesanal.

Hoy por hoy en todos los oficios artesanales se contempla el tema de materias primas; por lo menos existe la documentación de algunos aspectos relacionados, aunque aún no se hace un abordaje integral que comprenda todos los aspectos necesarios para fortalecer este eslabón de la cadena de valor. En algunos casos hay diagnósticos y protocolos de aprovechamiento de materias primas bien desarrolladas, pero al momento de hacer la implementación de acciones o planes de manejo, no se hacen actividades con la constancia y seguimiento necesario para que el uso y manejo sostenible se incorpore a las prácticas cotidianas de la población recolectora, cultivadora y artesana del caso. Adicionalmente, los componentes social y cultural son muy poco desarrollados, hay un mínimo trabajo en torno a las formas de asociatividad, ya sea con miradas convencionales (asociaciones, cooperativas, etc.) o con iniciativas locales de acuerdo a la propia cultura de artesanos, recolectores y/o cultivadores (procesos de auto-gobernanza). A pesar de los avances, se podría decir que lo más recurrente es que la población de recolectores y cultivadores de materias primas aún sigue siendo un eslabón muy débil del oficio artesanal, aunque cabe resaltar que ya no son invisibles en la mayoría de casos.

\section{Materias primas, uno de los soportes de los oficios artesanales}

Actualmente las materias primas son reconocidas como parte de los pilares de los oficios artesanales (Linares et al., 2008), sin duda son un componente indispensable en la conformación de las artesanías, de hecho, una artesanía se considera como tal de acuerdo al proceso y material con el que fue elaborada (UNESCO, 2017; Artesanías de Colombia, 2017). A medida que el rol de las materias primas ha sido más expuesto, se han identificado aspectos importantes que se deben tener en cuenta en la producción artesanal. La disponibilidad, calidad, formas de aprovechamiento, redes de comercialización y dinámicas de mercado como oferta/demanda/precio son, entre otros aspectos, los de mayor desarrollo investigativo en relación al tema. Las dinámicas alrededor de las materias primas suelen ser de forma muy espontánea, con el mínimo de intervención institucional, y se crean de acuerdo a las posibilidades de cada pueblo o comunidad, con todos los limitantes que esto implica, lo que genera distintas problemáticas según cada oficio artesanal.

\section{Tejeduría en caña flecha}

La tejeduría en caña flecha se hace a partir del uso de las fibras provenientes de Gynerium sagitatum (Aubl.) P.Beauv., una caña silvestre que actualmente es cultivada; su trabajo artesanal se remonta a las prácticas prehispánicas de los Zenú. Actualmente los Zenú reconocen tres variedades distintas de caña flecha, entre las que se encuentra la variedad conocida como "palma criolla", a la que se le atribuye la mejor calidad; esta variedad es cultivada y solo se encuentra dentro del resguardo Zenú de San Andrés de Sotavento (Casas, 2010).

Los indígenas Zenú y su territorio han recibido un fuerte apoyo institucional desde hace varias décadas. A partir de los años sesenta varias instituciones han destacado el trabajo artesanal en distintos proyectos, ya que es uno de los principales motores de la economía local.

A pesar del apoyo institucional y del tiempo que se lleva trabajando en la región, son muchas las carencias de la comunidad y las necesidades básicas de la población 
insatisfechas, hay problemas de seguridad alimentaria, el abastecimiento de agua es muy precario y no hay fuentes de empleo. Además, en lo que respecta a las artesanías, hay problemáticas identificadas desde hace varios años que todavía no están resueltas, lo que genera barreras en la producción que conllevan al estancamiento en el mejoramiento de la calidad de vida de artesanos y recolectores. Por ejemplo, por lo menos desde el 2003 (Artesanías de Colombia, 2003) se reportan los problemas de escasez de materia prima de "palma criolla" y el bajo pago que reciben los tejedores por su trabajo de elaboración de trenzas, dicha situación se mantiene en la actualidad (Casas, 2017c).

De hecho, Artesanías de Colombia S. A. ha identificado que: la regulación del mercado de la trenza, la generación de asociatividad entre trenzadores y la capacidad de acopio y regulación de la oferta de trenza; son indispensables para que los trenzadores perciban un mayor beneficio económico, por lo que sería muy interesante que se desarrollaran proyectos exitosos en torno a esto. Por la forma en la que está establecida la cadena de valor y la manera de vida de la comunidad en el resguardo, entre más etapas del proceso pueda hacer el mismo artesano, más beneficio económico va a obtener. Finalmente, es evidente que los procesos desarrollados para mejorar y fortalecer la producción de caña flecha no han sido exitosos, en gran medida porque se desarticulan del resto de la dinámica de los productores que se caracterizan por ser más campesinos que artesanos (Casas, 2017c) (figura 1 a,b,c).

\section{Cestería en esparto}

En el oficio de cestería en esparto se emplean las hebras de Juncus ramboi Barros, como materia prima, estas hebras provienen de una planta silvestre que únicamente se encuentra en los páramos de la cordillera oriental en Colombia (Lozano et al., 2008). El hecho de que la fibra venga de una planta silvestre y nativa hace que este producto se pueda enmarcar en los parámetros del Biocomercio y del aprovechamiento de Recursos Naturales No Maderables del bosque (PNNM); lo cual puede contemplar una diferenciación de mercados y una serie de requisitos legales que se deberían cumplir, tal como es el caso de la obtención de permisos de aprovechamiento para poder recolectar la fibra y transportarla libremente (Torres y Casas, 2014).

En este oficio se presentan problemas de abastecimiento, los recolectores han estado desvinculados de los proyectos y hasta hace poco se visibilizaron como parte importante 142 de la cadena de valor; no obstante, no se han contemplado en proyectos de la manera que se les permita organizarse y mejorar las dinámicas de abastecimiento, aspectos de su calidad de vida y algún tipo de autorreconocimiento de su trabajo como parte de la cadena de mercado de las artesanías en esparto.
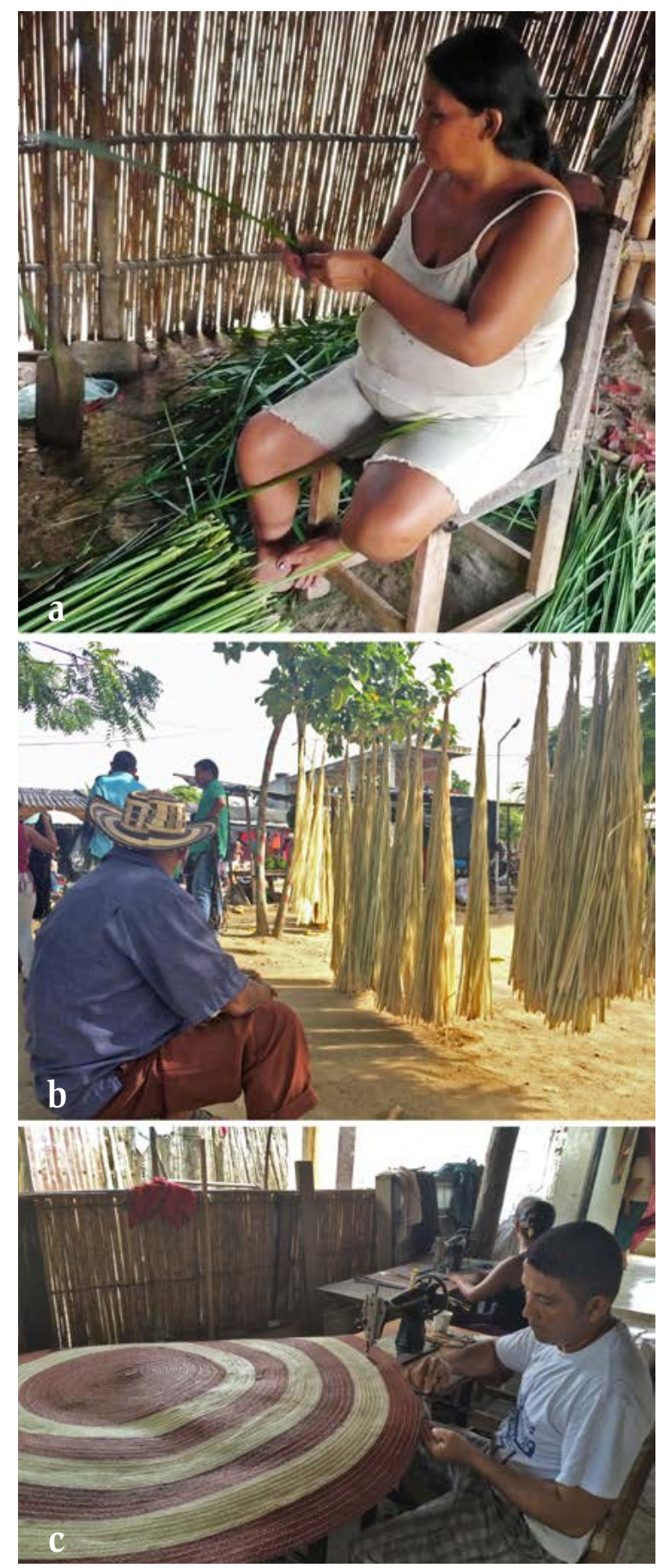

Figura 1. a) Recolectora procesando la caña flecha. b) Comerciante vendiendo la caña flecha lista para tejer y c) Artesano cosiendo un tapete, producto final. 
Un buen ejemplo de cómo los proyectos realizados por las distintas instituciones sí pueden llegar a tener un efecto en las dinámicas locales, tanto de mercado como de relacionamiento, es el caso de un proceso que inició Corpoboyacá de la mano de Artesanías de Colombia en el 2008 y 2009 (Lozano, 2009), en el que se fortaleció la cadena de valor del esparto al acercar a artesanas y recolectores. Como resultado de este proyecto, se hicieron reuniones en las que participaron artesanas y recolectores, donde se intercambiaron inquietudes, nombres y teléfonos. Como consecuencia de esta interacción, la venta de esparto cambió drásticamente en los últimos años. Anteriormente se vendía en Belén, Boyacá; para este evento de compra y venta las artesanas se desplazaban desde Cerinza y los recolectores desde el páramo del Güina. Aquí, el trato entre todos era hostil e incluso agresivo, había mucha competencia por la fibra y constantemente quedaban insatisfechos recolectores y artesanas (Casas, 2017a).

Los recolectores pocas veces lograban el precio que ellos consideraban justo y parte de las artesanas solían quedar desabastecidas de fibra y "perdían el viaje", pues muchas veces el esparto que llegaba al mercado no era suficiente para todas. El abastecimiento de la fibra era bastante variable, algunos sábados iban muchos recolectores y otros sábados no iba ninguno. Lo mismo ocurría con las artesanas, lo cual afectaba notablemente el precio y la calidad, pues el esparto se dañaba si se guardaba una semana más (Casas, 2017a).

Luego de los encuentros entre recolectores y artesanas, promovidos por el proyecto, con el tiempo la forma de compra y venta de esparto fue cambiando hasta el punto que hoy en día muchas de las artesanas tienen proveedores que llevan la fibra hasta sus casas; lo que ha mejorado las condiciones tanto para recolectores como para artesanas, pues el trato entre ellos es personalizado y de acuerdo a las posibilidades y necesidades de cada uno. Ya las artesanas no tienen que salir a pelear entre ellas a ver quién se queda con la materia prima, el precio lo arreglan entre recolector y artesana sin la presión de otros alrededor, y el recolector lleva la materia prima cuando tiene suficiente sin tener que guardarla durante mucho tiempo al punto de afectar su calidad. En definitiva, este cambio en la forma de venta del esparto ha tenido un impacto positivo en recolectores y artesanas (Casas, 2017a).

\section{Tejeduría en Wérregue}

En el caso del wérregue, se puede decir que es una materia prima que ha recibido una fuerte atención desde los procesos de fortalecimiento del sector artesanal; procesos en los que han intervenido muchas instituciones, desde hace aproximadamente tres décadas. Si bien el fortalecimiento comprende todos los eslabones de la cadena productiva, la materia prima ha sido protagonista en este caso.
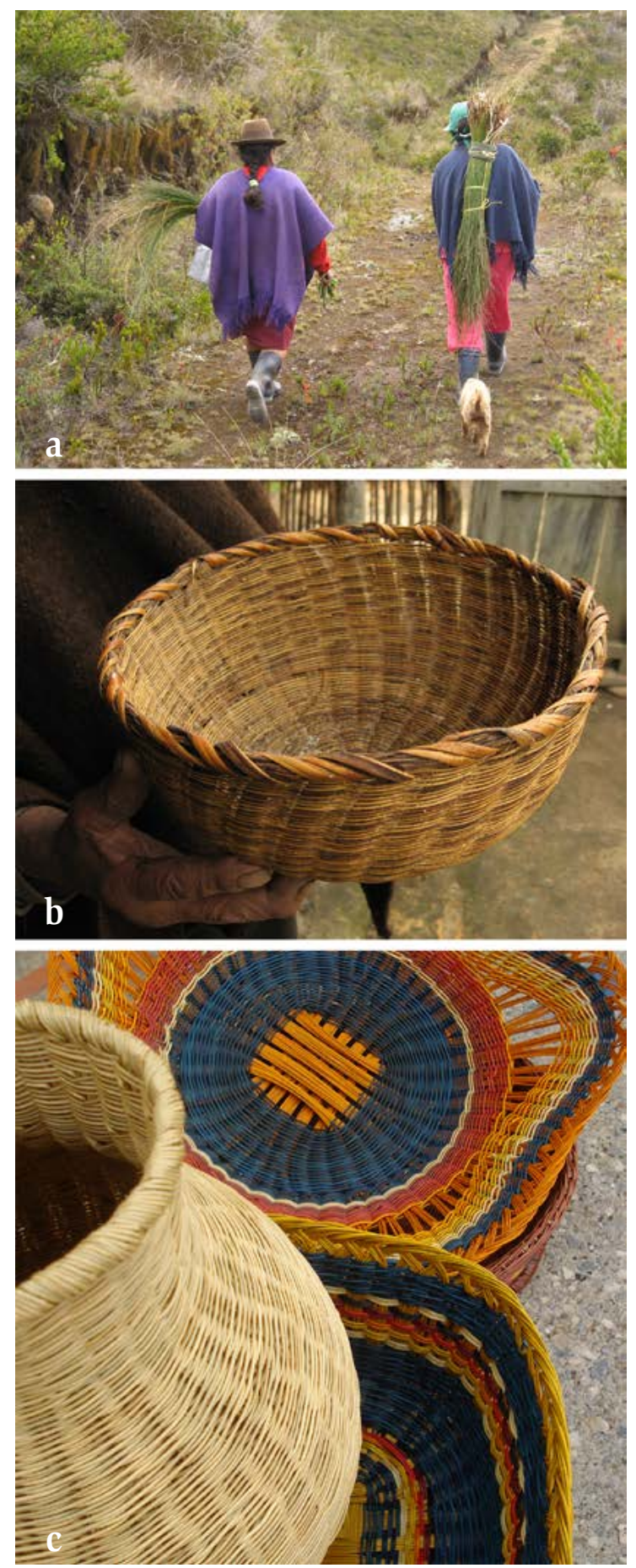

Figura 2. a) Recolectoras de esparto en el páramo. b) Colador en esparto, cesto tradicional y c) Cestos en esparto usando gama de colores. 
Las principales fibras usadas en la tejeduría con wérregue son: la palma de wérregue (Astrocaryum standleyanum L.H.Bailey) cuyas fibras están a la vista y envuelven fibras de otras plantas que se utilizan como relleno o alma del tejido; para el alma se usa la palma quitasol (Mauritiella macroclada (Burret) Burret), la palma amargo (Welfia regia $\mathrm{H}$. Wendl.) o la iraca (Carludovica palmata Ruiz \& Pav.). La cantidad de fibra que se usa tanto para el tejido como para el alma es semejante, razón por la cual es de igual importancia atender las necesidades de manejo de todas las plantas por igual y no solo la del wérregue. Con el fin de contrarrestar los problemas de escasez de fibra, varias instituciones han venido liderando procesos de manejo sostenible de la palma de wérregue, los cuales en algunos pocos casos se han extendido al manejo de la palma amarga y palma quitasol. Estos procesos han sido tan importantes que lograron involucrar de manera definitiva las actividades de manejo de la palma, y de ahí en adelante se han planteado en casi todos los proyectos de fortalecimiento. De hecho, en Colombia el wérregue puede ser considerado como el oficio artesanal con mayor desarrollo en el manejo sostenible de materias primas (Casas, 2017b).

Todas las materias primas empleadas en este oficio son de origen silvestre, además de ser especies nativas. La recolección de las fibras se hace en los bosques aledaños a las comunidades $y$, si bien existen varias iniciativas de propagación de estas especies para enriquecer bosques y solares de las casas, esto no es considerado como un cultivo en el sentido estricto, por lo que existen actualmente problemas de desabastecimiento e incluso extinciones locales (Casas, 2017b).

Es de resaltar que por medio de procesos como los que se han dado sobre el manejo de esta materia prima, se ha logrado llegar a acuerdos comunitarios sobre el manejo y uso de su territorio; abonando el terreno para entender y proyectar estos acuerdos de trabajo a otras comunidades artesanales del país (figura 3 a,b,c).

\section{Aplicación en tamo}

El oficio de aplicación en tamo suele hacerse con tamo de trigo y de cebada, materia prima que figura como un subproducto de los cultivos de estos cereales. Como el origen de este material es el cultivo, tiene implicaciones muy distintas a las que se evidencian en los ejemplos ya mencionados de los oficios artesanales en caña flecha, esparto y wérregue. Este caso permite evidenciar otros matices del papel de las materias primas en las cadenas 144 de valor y en la cultura de los artesanos (López, 2017).
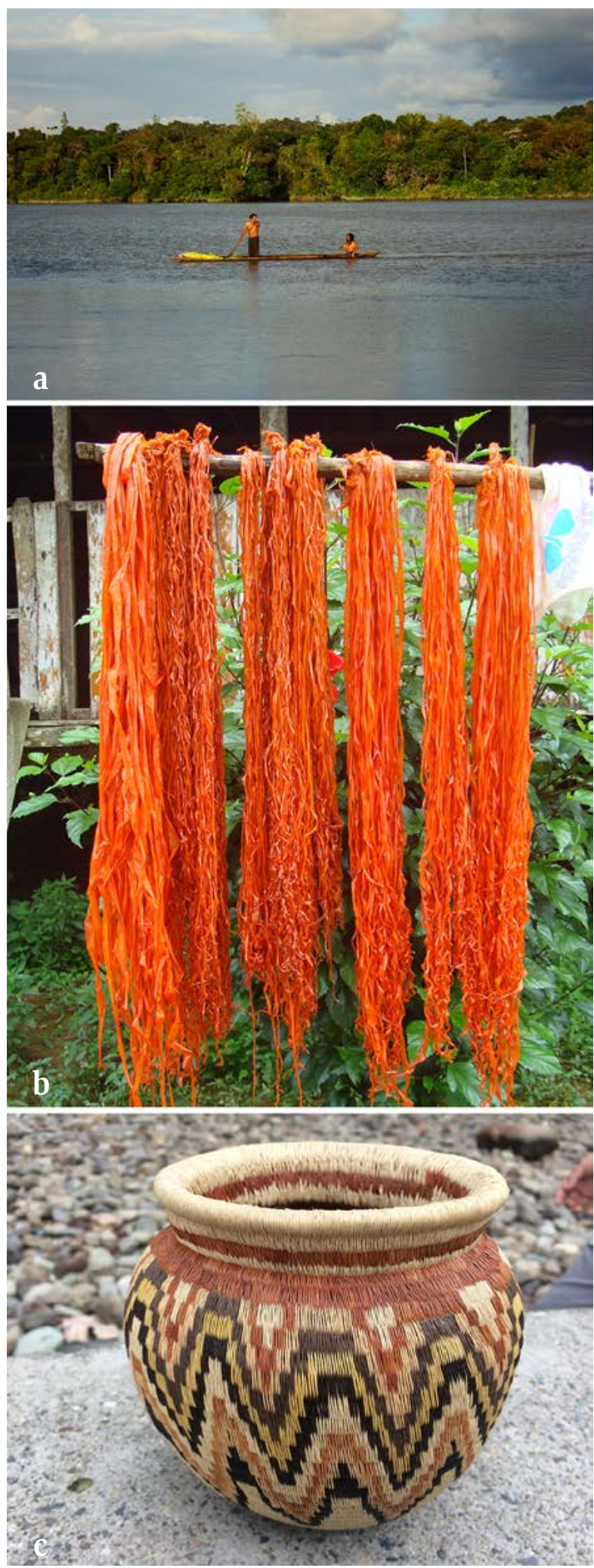

Figura 3. a) Artesanos transportando la fibra de wérregue. b) Fibra de wérregue secándose luego de haber sido teñida con tintes naturales y c) Canasto de wérregue para la venta. 
Al momento de revisar la información bibliográfica existente sobre este oficio, no se identificaba a los encargados de sacar el tamo y de transformarlo en láminas coloridas que compran los artesanos para hacer los enchapados. Esto deja de lado a un indispensable grupo de gente en la cadena productiva, no permite que ellos y su trabajo sea visible y que, por lo tanto, tampoco se puedan identificar sus carencias y necesidades, además de otras situaciones críticas como los cuellos de botella en la producción. En este caso sólo existe una familia que se encarga de laminar el tamo de trigo o cebada, lo cual sin duda constituye un punto crítico de la cadena de producción pues los miembros de esta familia son de avanzada edad y si ellos mueren o deciden dejar de hacer el trabajo de laminado no se sabe quién se encargaría de reemplazarlos, lo que generaría una ruptura en la cadena productiva y la consecuente escasez de láminas de tamo.

La situación de poca o nula visibilidad de uno o varios eslabones de la cadena productiva se asemeja a lo identificado en los oficios de tejeduría de caña flecha (cultivadores y trenzadores) y de esparto (recolectores); en este caso no se hacen visibles quiénes trabajan en las partes más básicas de la cadena de valor: laminadores y artesanos de talleres satélite (decoradores o entamadores). En el oficio de aplicación en tamo se ha dado un aumento en la demanda de materia prima y las dificultades para obtenerla han hecho que su precio haya aumentado hasta más del doble en los últimos 15 años. Este incremento en la demanda de materia prima, responde también a un incremento en el comercio de estas artesanías, talleres y artesanos (López, 2017).

Por otro lado, los cultivos de trigo y cebada que ofrecen el tamo para los artesanos están en declive (Machado, 2011). Los artesanos señalan que el tamo debe venir de pequeños cultivadores de trigo o cebada, ya que la recolección del tamo implica su cosecha manual con hoz o cuchillo, pues las máquinas segadoras despedazan la espiga del trigo y la cebada (López, 2017). La poca oferta de tamo llega a tal punto que en algunas ocasiones a los artesanos les ha tocado mandar a sembrar el tamo y crear un tipo de alianza con los cultivadores, que incluye préstamo de dinero. Algunos artesanos se han planteado contratar una siembra para todo el año (López, 2017).

Por último, es de resaltar que el enchapado en tamo se hace sobre piezas de madera, otra materia prima indispensable en el proceso de elaboración de estas artesanías; lo que acarrea sus propios problemas de abastecimiento y legalidad. De hecho, ante la desaparición de los árboles maderables en los bosques altoandinos alrededor de Pasto, los artesanos se han articulado con la cadena de extracción de maderas de los bosques húmedos de la Amazonía y del Pacífico. Además se han propuesto buscar alternativas maderables a través del uso de materiales aglomerados, así pueden solventar algunas de las dificultades biofísicas de las "maderas vivas" y de los requerimientos de las autoridades ambientales (López, 2017).

\section{Cestería en rollo de guacamayas}

La tejeduría de Guacamayas sobresale en cuanto al uso de materias primas, porque para la elaboración de los productos se emplean pajas de origen silvestre y nativo, así como de origen cultivado como el fique. Las pajas que se usan son las fibras para hacer el alma (la parte interna que da estructura a la pieza). De estas pajas se usan dos tipos: paja Blanca o lisa (Calamagrostis intermedia (J.Presl) Steud.) y paja de páramo o crespa (Calamagrostis effusa (Kunth) Steud.); además del uso del fique (Furcraea hexapetala (Jacq.) Urb.), esta última empleada para recubrir el alma y la fibra que queda expuesta y a la vista (Cárdenas, 2017).

La paja blanca era usada de manera generalizada entre los primeros artesanos, ya que se encontraba disponible en las zonas cercanas a las viviendas. Con el aumento en la demanda y de un manejo no adecuado, la disponibilidad de esta se redujo sustancialmente; así que durante la evolución de la artesanía en Guacamayas los artesanos identificaron que el rendimiento de la paja de páramo o crespa es mayor pues es mucho más larga y gruesa. Hoy son muy pocos los artesanos que usan la paja lisa; quienes todavía la utilizan es porque se autoabastecen porque está en sus predios. Se debe agregar que esta especie ha sido propagada, lo que permite un mejor abastecimiento, pero esta propagación no se ha realizado de manera técnica, de tal forma que se establezca como un cultivo en sentido estricto (Cárdenas, 2017).

Frente a esta situación, como iniciativa de la administración municipal, en el año 2014 se entregaron plantas de fique a los artesanos, para ser cultivadas y aprovechadas en la artesanía. Esta iniciativa no ha generado los resultados esperados, porque para los artesanos es más sencillo traer el fique del municipio de Curití en Santander y del municipio de Totoró en el Cauca (Cárdenas, 2017).

Generalmente resulta mejor fortalecer las cadenas para abastecerse de la materia prima de la calidad y cantidad requerida, que tratar de hacer que los artesanos desarrollen todos los eslabones de la producción. Esto es un tema que vale la pena analizar de acuerdo a cada caso. Es claro que 
la recolección y el cultivo para la producción de materia prima está más asociada a labores de campo que a las labores artesanales. En Colombia el fique es una de las fibras naturales con mayor desarrollo de tecnología y mercado (Linares et al., 2008), por lo que no vale la pena invertir esfuerzos en que en Guacamayas se produzca su propio fique; diferente es el caso cuando la producción de fique involucra un valor agregado a nivel cultural, tal como ocurre en el oficio artesanal de tejeduría de mochilas kankuamas.

\section{Cuellos de botella asociados a las materias primas}

A partir de las entrevistas semiestructuradas y el análisis de las materias primas empleadas en los distintos oficios artesanales, se identificaron diferentes cuellos de botella o barreras relacionadas con problemáticas de: abastecimiento, calidad, precio y organización de cultivadores o recolectores.

\section{Abastecimiento insuficiente}

De acuerdo con Artesanías de Colombia S. A., el 45,35 \% de la población artesanal dice sufrir de escasez de materias primas o de problemas asociados a la calidad 29,94\% (Artesanías de Colombia, 2001). Al hablar de un déficit de materia prima, lo primero que se suele pensar es en la escasez del recurso natural como tal, lo cual está asociado a factores ambientales; sin embargo, entre estos factores ambientales también hay situaciones importantes como los eventos climáticos extremos (veranos o inundaciones), las plagas, las tasas de crecimiento de las plantas de interés, las facilidades para propagarla, etc. Además de los aspectos ambientales, se hace énfasis en factores económicos y sociales, que pueden tener un fuerte efecto en la disponibilidad de materia prima. Las fibras usadas como materias primas en las artesanías son obtenidas a partir de plantas silvestres o cultivadas. Este hecho es determinante en la oferta natural de este recurso; pues si las plantas de interés se pueden cultivar, se presume que los problemas de abastecimiento se pueden solucionar más fácilmente, ya que se le puede dar manejo a la cantidad de producción requerida. No obstante, los aspectos sociales y económicos también son determinantes en la producción para definir la abundancia de la materia prima.

En el caso de las materias primas extraídas de plantas silvestres, la escasez puede deberse principalmente a una baja oferta natural, ya sea porque las poblaciones naturales de las plantas proveedoras son pequeñas o han disminuido, por destrucción del hábitat o por prácticas de uso y 146 aprovechamiento inadecuadas; pues si las materias primas son explotadas de forma más acelerada que su ritmo natural de crecimiento, no hay forma de compensar este déficit.

A nivel social y económico, encontramos casos en los que los problemas de abastecimiento se dan por el bajo nivel de organización de los recolectores o cultivadores y no por la falta de materia prima en sí. La disponibilidad de materia prima puede no ser constante, no porque las plantas de donde se extraen tengan una oferta natural restringida 0 porque su producción solo sea en temporadas específicas, sino porque los ritmos de recolección o cosecha dependen de otros factores como el precio de la materia prima en relación con otros productos. Generalmente, la recolección de materias primas de origen silvestre para uso artesanal, hace parte de actividades económicas complementarias de los recolectores y estos dejan de realizarla cuando encuentran mejor remuneración en otras actividades.

\section{Calidad}

La calidad de la materia prima también es un factor importante pues, en parte, de esta va depender la calidad del producto final. Una buena o mala calidad va a depender de las características propias de las plantas o variedades de estas que sean recolectadas o cosechadas, pero además entran a jugar otros factores como: buenas prácticas de recolección y cosecha, conocimiento sobre las técnicas de extracción, tiempo adecuado para la cosecha o recolección (edad de la planta, clima, luna, etc.), tiempos de transporte y condiciones de almacenamiento, entre otros.

Para resolver muchos de los problemas que limitan la calidad, hace falta mayor fortalecimiento de la cadena de valor, pues en la medida en la que se dificulten o faciliten los procesos, la calidad puede mejorar. Por ejemplo, es indispensable fortalecer los temas asociados a cultivos, acuerdos para realizar la recolección en áreas y condiciones adecuadas, medios de transporte, adecuación de sitios para el almacenamiento, facilitación de la transmisión de conocimiento sobre manejo, extracción de fibras, etc.

\section{Precio}

El precio afecta de manera diferente a recolectores o cosechadores y artesanos. Los recolectores o cosechadores frecuentemente mencionan que el pago que reciben por su trabajo es muy poco, con relación al esfuerzo que hacen; mientras que los artesanos consideran que el precio de la materia prima es alto. Una forma de disminuir la brecha que existe en la percepción de estos dos grupos puede ser la de socializar la importancia y las dificultades que enfrentan cada uno, así se genera más conciencia del valor 
del trabajo que a cada uno le corresponde. La falta de un abastecimiento constante influye directamente en el precio, de manera similar a cualquier dinámica comercial; en muchos casos estas dinámicas pueden ser estabilizadas por medio del correcto fortalecimiento de la cadena de valor.

\section{Nivel de organización y otras dinámicas sociales}

El nivel de organización de los recolectores o cultivadores resulta ser indispensable para lograr un abastecimiento constante y suficiente, pues un sólido nivel de organización les podría permitir tener una capacidad de respuesta, de acuerdo a la demanda de materia prima por parte de los artesanos. Adicionalmente, entre las personas que conforman estos eslabones de la cadena productiva, se podrían fortalecer las redes de comercialización y comunicación. Es indispensable que todos los eslabones de la cadena sean igual de visibles, para que puedan ser involucrados en los proyectos y programas de fortalecimiento, sin excluir a ninguno, y que se atienden a problemáticas y necesidades reales y no solo desde la mirada institucional.

A nivel social también ocurren otros eventos que afectan de cierta forma las capacidades de trabajo en todos los niveles; se trata de problemas de manejo territorial, celos y rencillas y en muchos casos pérdida de conocimiento sobre el manejo de ciclos asociados a cultivos, recolección y extracción.

Por otro lado, existen problemáticas propias de la asistencia institucional que se brinda a las comunidades a nivel organizacional; generalmente las intervenciones institucionales son inconclusas, incapaces de mantener a largo plazo procesos con las comunidades, debido a falta de visión, de presupuesto o por las propias dificultades de acceso continuo a ciertos territorios. A esto se le suma la incapacidad de generar condiciones para el desarrollo del oficio que sean autosostenibles a lo largo del tiempo. En estos procesos de apoyo institucional se han aplicado metodologías lejanas a los contextos propios de los recolectores, cultivadores o artesanos en medio de escenarios con fuerte abandono del campo, problemas de seguridad alimenticia y problemas de violencia y orden público, entro otros.

\section{Manejo sostenible de materias primas, de las generalidades al estudio de caso}

El interés en la innovación ambiental, o cambio de conductas ambientales, por parte del negocio de las artesanías, es un fenómeno relativamente reciente
(Sánchez-Medina, 2011), a pesar de ser indispensable en el caso de las materias primas de los oficios artesanales, pues este tipo de manejo es fundamental para continuar con el crecimiento del sector artesanal a largo plazo. El manejo sostenible debe tener un equilibro con los aspectos sociales y económicos, ya que es la única manera en la que las prácticas de manejo pueden llegar a ser realidad y permanecer en el tiempo (Leakey et al., 1996). Es de resaltar que este tipo de manejo, además de buscar el abastecimiento, busca evitar la extinción local de las especies proveedoras de materias primas, la repartición justa de ingresos y fomentar el uso de la biodiversidad (Estrategia Nacional para la Conservación de Plantas, 2001).

\section{El wérregue como caso de estudio}

En el caso de la palma de wérregue, se pasó de la falta de interés y los vacíos de información a acciones como la elaboración de planes de manejo sostenible, elaboración y entrega de herramientas de corte, enriquecimiento de predios con plántulas de wérregue y, posteriormente, también un interés en el manejo y conservación de palma quitasol y palma amarga, las otras materias primas asociadas a este tejido. En el momento es uno de los oficios artesanales que más desarrollo ha tenido en el manejo sostenible, situación que tuvo su inicio como respuesta a problemas de abastecimiento. Con el fin de contrarrestar la escasez, los efectos negativos ambientales y abrir más el mercado, varias instituciones (públicas, privadas y ONGs) han venido liderando procesos de manejo sostenible de la palma de wérregue, los cuales en algunos pocos casos se han extendido al manejo de la palma amarga y el quitasol.

Estas iniciativas se han dado en varias comunidades y han tenido alcances como: la creación de planes de manejo y aprovechamiento sostenible (son acuerdos para el manejo de la palma en una comunidad puntual), protocolos de aprovechamiento sostenible (en estos se dan pautas generales de manejo que aplican para todas las comunidades), siembras con fines de repoblamiento, creación de herramientas de corte (medialuna) con características de tamaño y formas específicas para las palmas de wérregue, además de variado material de apoyo para las comunidades (cartillas, videos, plegables, etc.) (Lozano, 2012; García et al., 2011; Casas, 2017b).

Dado que las iniciativas de manejo sostenible del wérregue han sido las más recurrentes, en muchas de las comunidades la gente cuenta con estas palmas sembradas en sus parcelas, ya sea como resultado de los procesos 
institucionales o por iniciativa propia. Ocasionalmente se han dado siembras de palma quitasol y de amargo por cuenta de los pobladores y han logrado experiencias exitosas (Lozano, 2012). Esto ejemplifica el nivel de apropiación y comprensión de la dinámica de los recursos por parte de artesanos del wérregue.

El uso de la medialuna para el aprovechamiento de cogollo de wérregue es extendido entre varias comunidades del Bajo San Juan. Según los mismos habitantes esto ha sido un proceso de varios años hasta que llegaron a habituarse a su uso. Las primeras iniciativas para emplear las medialuna fueron las realizadas por Artesanías de Colombia S.A. y la FES en 1994 y 1995. El proceso y la apropiación del manejo de la medialuna también ha resultado en el uso de esta herramienta para el aprovechamiento de cogollos (hojas jóvenes) de otras palmas, como el quitasol y el amargo; sin embargo, el quitasol aún es intensamente derribado para la obtención del cogollo. Las prácticas de manejo sostenible ya se encuentran incorporadas en la cotidianidad de la población indígena, pero aún es indispensable incluir a las comunidades negras en los proyectos y programas que se realizan, pues ellos cortan grandes cantidades de cogollos y no poseen medialunas, por lo que tumban muchas palmas (Torres, 2010; Lozano, 2012).

\section{Conclusiones y recomendaciones}

Se hace evidente que fortalecer el abastecimiento y calidad de materia prima promueve la cadena de valor artesanal y brinda oportunidades de mercado valiosas. Es importante entender que no necesariamente los artesanos tienen una relación directa con el abastecimiento de las materias primas, así que resulta relevante comprender las cadenas de abastecimiento en cuanto a calidad y cantidad requerida. Asimismo, se resalta que casi nunca la solución a los problemas de abastecimiento es hacer que los artesanos desarrollen cultivos o hagan recolección de materia prima; esto es un tema que vale la pena analizar caso por caso, identificando claramente los eslabones en los que participan artesanos y recolectores y diferenciando que, generalmente, la producción de materia prima (recolección y cultivo) está más asociada a labores de campo que a labores artesanales.

En términos ambientales, es muy importante entender las características propias de cada planta y su aprovechamiento con el fin de saber cómo se pueden ajustar a los requerimientos artesanales y de mercado sin generar una amenaza para la conservación de las especies. La 148 investigación académica para la comprensión de las dinámicas ecológicas y de producción de materias primas es deseable y debe ser blanco de acción de universidades y centros de investigación. Los resultados de la investigación aportan un mejor manejo. Además, en muchos casos existen buenas prácticas de aprovechamiento y manejo de las materias primas realizadas por los recolectores quienes, basados en su experiencia, crean formas de manejo compatibles con la sostenibilidad.

De manera integral, desde los temas ambientales se llega a procesos sociales importantes en las comunidades, donde en el mejor de los casos se generan mecanismos de autogobernanza que tienden a dar un manejo sostenible a las materias primas. El proceso de incorporación de prácticas de uso y aprovechamiento de materias primas debe tener acompañamiento institucional, pero debe tender a procesos autónomos y no a la dependencia del asistencialismo institucional.

Se deben involucrar todos los eslabones de las cadenas de valor en los proyectos de fortalecimiento al sector artesanal; es decir, desde los involucrados en la siembra o recolección de materias primas, en la transformación y la obtención del producto final. Dejar por fuera cualquiera de estos eslabones significa desventajas sociales para quienes trabajan en él, pues se vuelven invisibles y esta invisibilidad se perpetúa a nivel institucional; tal como sucedió durante décadas con los recolectores o cultivadores de materias primas. Sin embargo, con el cambio de paradigmas que ha traído el concepto de sostenibilidad en el oficio artesanal, es cada vez más frecuente en la agenda de programas y proyectos del sector artesanal la inclusión de todo lo relativo al abastecimiento de materias primas.

Cada oficio artesanal tiene diferentes problemáticas en cuanto a las materias primas, pueden ser de orden ambiental, social o de legalidad. Para cada caso se deben evidenciar sus particularidades y, como se discute en este documento, abordarse de acuerdo a estas para buscar soluciones. En general, se puede decir que los problemas relacionados con materias primas no son situaciones aisladas y se deben tener en cuenta las dinámicas ambientales, sociales y económicas al momento de fortalecer las cadenas de valor, pues de esto puede depender el éxito o no de los proyectos propuestos.

\section{Agradecimientos}

Este artículo surge como resultado del proyecto "Saberes, significados y mercados. Análisis crítico sobre la transformación en el oficio artesanal en Colombia”, 
liderado por la Fundación Erigaie con acompañamiento de la Fundación Natura, gracias a la financiación de Colciencias y Artesanías de Colombia S.A. 2016-2018. Agradecemos a todos los artesanos, recolectores y cultivadores de los diferentes oficios artesanales investigados.

\section{Referencias}

Artesanías de Colombia. 1998. Censo Económico Nacional del Sector Artesanal. Ministerio de Desarrollo Económico. Santafé de Bogotá, Colombia.http://repositorio.artesaniasdecolombia. com.co/handle/001/1835. Consultado: 18 de noviembre 2017.

Artesanías de Colombia. 2017. El sector artesano en Colombia. Ministerio de Industria y Turismo. Bogotá, http://www. artesaniasdecolombia.com.co/PortalAC/C_sector/ la-artesania-y-su-clasificacion_82. Consultado: 18 de noviembre 2017.

Artesanías de Colombia. 2003. Presentación institucional de la cadena productiva de la caña flecha, departamentos Córdoba y Sucre. Ministerio de Industria y Turismo. Bogotá D.C., Colombia. http://repositorio.artesaniasdecolombia.com. co/handle/001/2907. Consultado: 18 de noviembre 2017.

Artesanías de Colombia. 2001. Habilitación, uso y manejo sostenible de materias primas vegetales y de ecosistemas relacionados con la producción Artesanal en Colombia. Ministerio de Industria y Turismo, Bogotá D.C., Colombia.

Bernal, R. y G. Galeano. 2013. Cosechar sin destruir Aprovechamiento sostenible de palmas colombianas. Facultad de Ciencias-Instituto de Ciencias Naturales. Universidad Nacional de Colombia, Bogotá D.C.

Cárdenas, F. 2017. Informe de cestería en rollo, Guacamayas. Proyecto "Saberes, significados y mercados. Análisis crítico sobre la transformación en el oficio artesanal en Colombia". Fundación Erigaie, Bogotá D.C.

Casas, L.F. 2017a. Informe de tejeduría en caña flecha. Proyecto "Saberes, significados y mercados. Análisis crítico sobre la transformación en el oficio artesanal en Colombia". Fundación Erigaie, Bogotá D.C.

Casas, L.F. 2017b. Informe de cestería en esparto. Proyecto "Saberes, significados y mercados. Análisis crítico sobre la transformación en el oficio artesanal en Colombia”. Fundación Erigaie, Bogotá D.C.

Casas, L.F. 2017c. Informe de tejeduría en Wérregue. Proyecto "Saberes, significados y mercados. Análisis crítico sobre la transformación en el oficio artesanal en Colombia”. Fundación Erigaie, Bogotá D.C.
Casas, L.F. 2010. Cartilla para la producción sostenible de artesanías en caña flecha. Artesanías de Colombia. Ministerio de Industria y Turismo, Bogotá D.C.

Castaño, N., Cárdenas, D.L. y Otavo, E. 2007. Ecología, aprovechamiento y manejo sostenible de nueve especies de plantas del departamento del Amazonas, generadoras de productos maderables y no maderables. Instituto amazónico de investigaciones científicas-Sinchi, Bogotá D.C.

D`Amato, D., Droste, N., Allen, B., Kettunen, M., Lahtinen, K., Korhonen, J., Leskinen, P., Matthies, B.D. y Toppinen A. 2017. Green, circular, bioeconomy: A comparative analysis of sustainability avenues. Journal of Cleaner Production 168: 716-734.

Elzen, B., Geels, F.W. y Green, K. 2004. Theoretical explorations of transitions. In System Innovation and the Transition to Sustainability: Theory, Evidence and Policy. Edward Elgar Publishing Limited, Northampton, USA.

Estrategia Nacional para la Conservación de Plantas 2001. Instituto de investigación de recursos biológicos IAvH. Bogotá D.C. Colombia. http://www.humboldt.org.co/ images/documentos/pdf/investigacion/2001-estrategianacional-para-la-conservacin-de-plantas.pdf. Consultado: 18 de noviembre 2017.

Fondo Nacional para Fomento de las Artesanías, 2016. Secretaría de cultura. https://www.gob.mx/cultura/ documentos/cuenta-satelite-de-la-cultura-de-mexico. Consultado: 2 de agosto 2018.

García, N., Galeano, G., Mesa, L., Castaño, N., Balslev, H., and Bernal, R. 2015. Management of the palm Astrocaryum chambira Burret (Arecaceae) in northwest Amazon. Acta Botanica Brasilica 29(1): 45-57.

García, N., Galeano, G., Bernal, R. y Balslev, H. 2013. Management of Astrocaryum standleyanum (Arecaceae) for Handicraft Production in Colombia. Ethnobotany Research and Applications 11: 085-101.

García, N., Torres, M.C., Bernal, R., Galeano, G. Valderrama, N. y Barrera, V.A. 2011. Management of the spiny palm Astrocaryum malybo in Colombia for the production of mats. Palms 55(4): 190-195.

González, D. V. 2003. Los productos naturales no maderables (PNNM): Estado del arte de la investigación y otros aspectos. Biocomercio sostenible. Instituto de investigaciones biológicas Alexander von Humboldt, Bogotá D.C.

Haro-Zea, K., Haro-Zea, Y., Roblero-Mazariegos, G. y Sánchez, S. 2018. Chiapaneca Handicraft as a Driver of Sustainable 
Local Development. Global Conference on Business and Finance Proceedings 13(1):89-97.

Isaza, C., Bernal, R., y Howard, P. 2013. Use, Production and Conservation of Palm Fiber in South America: A Review. Journal of Human Ecology 42(1): 69-93

Kar, S.P. y M.G. Jacobson. 2012. NTFP income contri- bution to household economy and related socio-eco- nomic factors: Lessons from Bangladesh. Forest Policy and Economics 14(1):136-142.

Leakey, R.R.B, Temu, A.B., Melnyk, M. y Vontomme, P. 1996. Domestication and commercialization of non-timber forest products in agroforestry systems. FAO, Food and Agriculture Organization of the Unated Nations, Roma.

Linares, E. L., Galeano, G., García, N. y Figueroa, Y. 2008. Fibras vegetales empleadas en artesanías en Colombia. Instituto de ciencias Naturales-Universidad de Colombia, Bogotá D.C.

López, F. 2017. Informe de aplicación en tamo. Proyecto "Saberes, significados y mercados. Análisis crítico sobre la transformación en el oficio artesanal en Colombia”. Fundación Erigaie, Bogotá D.C.

Lozano, A. 2012. Comunidad Papayo, municipio Litoral del San Juan, departamento de Chocó. Etnia Wounan Artesanías de Colombia. Convenio DHS No 5211057 suscrito entre Ecopetrol S.A. Y Artesanías De Colombia S.A. Informe de actividades: Ministerio de Industria y Turismo, Bogotá D.C.

Lozano, A. 2009. Apoyo técnico para el aprovechamiento sostenible y legalización ambiental del esparto, Juncus ramboi, utilizado como materia prima para la elaboración de artesanías en el municipio de Cerinza, departamento de Boyacá. Informe. Artesanías de Colombia, Ministerio de Industria y Turismo - Corpoboyacá. Bogotá D.C.

Lozano A., Casas, L.F., Ospina, A.P. y Ramírez, D. 2008. Protocolo para la producción sostenible de artesanías en esparto Junus ramboi, departamento de Boyacá. Artesanías de Colombia, Ministerio de Industria y Turismo. Bogotá D.C.

Machado, A. 2011. Informe de desarrollo humano sobre desarrollo rural y tierras. Bogotá: Programa de las Naciones Unidas para el Desarrollo (PNUD). Bogotá D.C.

Marshall, E., Schreckenberg, K. Y Newton, A.C. 2006. Commercialization of Non-Timber Forest Products: Fac- tors in uencing success. Lessons learned from Mexico and Bolivia and policy implications for decision-makers. United Nations Environment Programme, World Conser-vation Monitoring 150 Centre, Cambridge.
Mekonnen, Z., Worku, A.R., Yohannes, T., Alebachew, M., Teketay, D. y Kassa, H. 2014. Bamboo Resources in Ethiopia: Their value chain and contribution to livelihoods. Ethnobotany Research \& Applications 12: 511-524.

Ministerio de Ambiente y Desarrollo Sostenible. 2014. Plan Nacional de Negocios Verdes. http://www.minambiente. gov.co/images/NegociosVerdesysostenible/pdf/plan_ de_negocios_verdes/Plan_Nacional_de_Negocios_ Verdes.pdf. Consultado: 18 de noviembre 2018.

Moraes, I. 2010. Design aplicado ao artesanato, uma ferramenta para a sustentabilidade: estudo de caso sobre a comunidade de Nossa Senhora do Perpétuo Socorro de Acajatuba, município de Iranduba/am. Tesis de maestría, Universidade Federal do Amazonas, Manaos, Amazonas, Brasil.

PGNGIBSE, 2012. Política Nacional para la Gestión Integral de la Biodiversidad y sus Servicios Ecosistémicos. Ministerio de Ambiente y Desarrollo Sostenible, Bogotá D.C.

Programa Nacional de Biocomercio Sostenible. 2014. Programa de desarrollo biocomercio sostenible 2014-2024. Ministerio de Ambiente y Desarrollo Sostenible, Bogotá D.C.

Ramírez, D. 2012. Notas sobre la historia de Artesanías de Colombia S.A. y la artesanía colombiana. Ministerio de Industria y Turismo, Bogotá D.C

Régimen aprovechamiento forestal 1996, Decreto 1791. Ministerio del Medio Ambiente. Bogotá D. C. Colombia.

Sachs, I. 2002. Desenvolvimento Sustentável, Bioindustrialização Descentralizada e Novas Configurações Rural-urbanas. En: Vieira, P.F. y Weber, J. Editores. Gestão de Recursos Naturais Renováveis e Desenvolvimento: Novos desafios para a pesquisa ambiental. Cortez São Paulo.

Sánchez-Medina, P., Corbett, J. y Toledo-López, A. 2011. Environmental Innovation and Sustainability in Small Handicraft Businesses in Mexico. Sustainability 3: 984-1002.

Torres M. C. 2010. Protocolo de aprovechamiento y estimación de la oferta para las especies de uso artesanal tetera (Stromanthe jacquinii) y chocolatillo (Ischnosiphon arouma) en el resguardo indígena de Joaquincito (Bajo Naya) y werregue (Astrocaryum standleyanum) en el resguardo indígena de Puerto Pizario (Bajo San Juan), Buenaventura, Valle del Cauca. Convenio 052 de 2008, Corporación Autónoma Regional del Valle del Cauca y Fundación Zoológica de Cali. Cali.

Torres, C., Galeano, G. y Bernal, R. 2016. Cosecha y manejo de Copernicia tectorum (Kunth) Mart. para uso artesanal en el Caribe colombiano. Colombia Forestal 19(1): 5-22. 
Torres, M.B. y Casas, L.F. 2014. Protocolos de aprovechamiento para la flora silvestre no maderable. Metodología, estudios de caso y recomendaciones técnicas. Fondo de Biocomercio - Fundación Natura, Bogotá D.C.
UNESCO.2017 Artesanía y diseño. http://www.unesco.org/ new/es/santiago/culture/creative-industries/crafts-design/ Consultado: 10 de noviembre 2017.

Citar como: Casas-Caro, L.F. y Lozano, A. 2018. Biocomercio y sostenibilidad: análisis en torno a las materias primas de las artesanías en Colombia. Intropica 13(2): 137-151. DOI: http://dx.doi.org/10.21676/23897864.2560. 